\title{
THE NACHBIN QUASI-UNIFORMITY OF A BI-STONIAN SPACE
}

\author{
P. FLETCHER, J. FRITH, W. HUNSAKER and A. SCHAUERTE
}

(Received 4 April 1994; revised 21 April 1995)

Communicated by J. A. Hillman

\begin{abstract}
It is known that every frame is isomorphic to the generalized Gleason algebra of an essentially unique bi-Stonian space $(X, \sigma, \tau)$ in which $\sigma$ is $T_{0}$. Let $(X, \sigma, \tau)$ be as above. The specialization order $\leq_{\sigma}$ of $(X, \sigma)$ is $\tau \times \tau$-closed. By Nachbin's Theorem there is exactly one quasi-uniformity $\mathscr{U}$ on $X$ such that $\cap \mathscr{U}=\leq_{\sigma}$ and $\mathscr{T}\left(\mathscr{U}^{*}\right)=\tau$. This quasi-uniformity is compatible with $\sigma$ and is coarser than the Pervin quasi-uniformity $\mathscr{P}$ of $(X, \sigma)$. Consequently, $\tau$ is coarser than the Skula topology of $\sigma$ and coincides with the Skula topology if and only if $\mathscr{U}=\mathscr{P}$.
\end{abstract}

1991 Mathematics subject classification (Amer. Math. Soc.): primary 54E15, 54E05, 54F05; secondary 06D20, 03G10.

\section{Introduction}

According to [4], a subset $G$ of a bitopological space $(X, \sigma, \tau)$ is strictly regular provided that $G=\operatorname{int}_{\sigma} \mathrm{cl}_{\tau} G$ and a bitopological space $(X, \sigma, \tau)$ is bi-Stonian provided that $\tau$ is a compact Hausdorff 0 -dimensional topology containing $\sigma, \sigma$ has a base of strictly regular sets and each strictly regular set is $\tau$-closed. The generalized Gleason algebra of a bi-Stonian space $(X, \sigma, \tau)$ is the frame of its strictly regular subsets, where $A \wedge B$ is defined as $A \cap B$ and $\bigvee A_{\alpha}$ is defined as int $\operatorname{cl}_{\tau} \cup A_{\alpha}$. The principal result of [4] is that every frame is isomorphic to the generalized Gleason algebra of an essentially unique bi-Stonian space in which the coarser topology is $T_{0}[4$, Theorem 3.2].

In this paper we consider only those bi-Stonian spaces $(X, \sigma, \tau)$ for which $\sigma$ is a $T_{0}$ topology. Under this restriction, as is well known, the specialization order of $\sigma$ is a partial order, and a simple lemma establishes that this partial order is $\tau \times \tau$-closed. Consequently, there is exactly one quasi-uniformity on $X$, which we call the Nachbin

(C) 1996 Australian Mathematical Society 0263-6115/96 \$A2.00+0.00 
quasi-uniformity and denote by $\mathscr{N}$, such that $\bigcap \mathscr{N}$ is the specialization order of $\sigma$ and $\mathscr{T}\left(\mathscr{N}^{*}\right)=\tau$ [3, Proposition 13] and [2, Theorem 1.20]. We show that $\mathscr{N}$ is compatible with $\sigma$ and that $\mathscr{N}$ is coarser than the Pervin quasi-uniformity of $(X, \sigma)$. It follows that $\tau$ is coarser than the Skula topology of $\sigma$ [6], and we show that $\tau$ is the Skula topology of $\sigma$ if, and only if, the Nachbin quasi-uniformity and the Pervin quasi-uniformity of $(X, \sigma)$ coincide. Moreover, there is a natural base $\mathscr{B}$ for $\mathscr{N}$ such that for each $U \in \mathscr{B}$ and $x \in X, U(x)$ is $\tau\left(\mathscr{N}^{-1}\right)$-closed and hence strictly regular and $\tau$-closed.

We refer the reader to [2] for definitions and results concerning quasi-uniformities that are assumed here.

\section{The Nachbin quasi-uniformity}

Throughout this paper, we consider a given bi-Stonian space $(X, \sigma, \tau)$ for which $\sigma$ is a $T_{0}$ topology. The specialization order of $\sigma$, which is denoted by $\leq_{\sigma}$, is defined by $x \leq_{\sigma} y$ if, and only if, $x \in \mathrm{cl}_{\sigma}\{y\}$. This order is a partial order.

LEMMA 2.1. The specialization order of $\sigma$ is $\tau \times \tau$-closed.

Proof. Suppose that $x \notin \operatorname{cl}_{\sigma}\{y\}$ and let $G$ be a strictly regular set about $x$ such that $y \notin G$. Then $(x, y) \in G \times(X-G)$, which is $\tau \times \tau$-open, and since $G \in \sigma$, it is evident that $\leq_{\sigma}$ and $G \times(X-G)$ are disjoint.

PROPOSITION 2.1. The Nachbin quasi-uniformity of $(X, \sigma, \tau)$ is compatible with $\sigma$.

PROOF. Let $\mathscr{B}$ be the base for $\sigma$ consisting of all strictly regular sets and let $B \in \mathscr{B}$. Then $B$ is $\tau$-open and $\tau$-closed and so $U_{B}=(B \times B) \cup((X-B) \times X)$ is a $\tau \times \tau$-open set containing $\leq_{\sigma}$. It follows that $U_{B} \in \mathscr{N}$ [3, Proposition 13], or [2, Theorem 1.20]. Let $\mathscr{V}$ be the quasi-uniformity on $X$ for which $\left\{U_{B}: B \in \mathscr{B}\right\}$ is a transitive subbase. It is evident that $\mathscr{V}$ is compatible with $\sigma$-we complete the proof by showing that $\mathscr{V}$ is the Nachbin quasi-uniformity. Since $\mathscr{V} \subseteq \mathscr{N}, \leq_{\sigma}=\bigcap \mathscr{N} \subseteq \bigcap \mathscr{V}$. Let $(x, y) \in \bigcap \mathscr{V}$. Then for each $B \in \mathscr{B},(x, y) \in U_{B}$ and so $x \in \operatorname{cl}_{\sigma}\{y\}$. Hence $\cap \mathscr{V}$ is $\leq_{\sigma}$. To see that $\mathscr{T}\left(\mathscr{V}^{*}\right) \subseteq \tau$, note that since $\mathscr{V} \subseteq \mathscr{N}, \mathscr{T}\left(\mathscr{V}^{*}\right) \subseteq \mathscr{T}\left(\mathscr{N}^{*}\right)=\tau$. Since $\sigma$ is a $T_{0}$ topology, $\mathscr{T}\left(\mathscr{V}^{*}\right)$ is a Hausdorff topology and so $\mathscr{T}\left(\mathscr{V}^{*}\right)=\tau$.

DEFINITION [6]. The Skula topology of a topological space $(S, \mathscr{G})$ is the topology on $S$ for which $\mathscr{G} \cup\{X-G: G \in \mathscr{G}\}$ is a subbase.

COROLlaRY 2.1. The Nachbin quasi-uniformity of $(X, \sigma, \tau)$ is a transitive quasiuniformity coarser than the Pervin quasi-uniformity of $(X, \sigma)$ and $\tau$ is coarser than the Skula topology of $\sigma$. 
ProOf. Let $\mathscr{P}_{\sigma}$ denote the Pervin quasi-uniformity for $(X, \sigma)$. The collection $\left\{U_{B}: B \in \mathscr{B}\right\}$ given in the proof of Proposition 2.1 is a subcollection of $\mathscr{P}_{\sigma}$ consisting of transitive entourages. Thus $\mathscr{N}$ is a transitive quasi-uniformity coarser than $\mathscr{P}_{\sigma}$. By [1, Proposition 1.4] and [5, Proposition 3.2.2.3], $\mathscr{T}\left(P_{\sigma}^{*}\right)$ is the Skula topology of $\sigma$ and so $\tau=\mathscr{T}\left(\mathscr{N}^{*}\right)$ is coarser than the Skula topology of $\sigma$.

Suppose for the nonce that $\sigma$ is a $T_{1}$ topology. Then $\leq_{\sigma}$ is the diagonal and so $\mathscr{N}$ is the only uniformity compatible with $\tau$. By the previous proposition, $\sigma=\mathscr{T}(\mathscr{N})=\tau$ and the frame corresponding to $(X, \sigma, \tau)$ is the Boolean algebra of $\tau$-regular open sets. This observation is an instance of a general principle: interesting topologies are always $T_{0}$ and never $T_{1}$.

PROPOSITION 2.2. Let $\mathscr{B}$ be a transitive base for the Nachbin quasi-uniformity of $(X, \sigma, \tau)$. Then for each $U \in \mathscr{B}$ and each $x \in X, U(x)$ is $\mathscr{T}\left(\mathscr{N}^{-1}\right)$-closed and $U^{-1}(x)$ is $\mathscr{T}(\mathscr{N})$-closed; hence both $U(x)$ and $U^{-1}(x)$ are $\tau$-closed, $\tau$-open and strictly regular.

PROOF. Let $U \in \mathscr{B}$ and $x \in X$. Then $\left\{U^{-1}(y): y \notin U(x)\right\} \bigcup\{U(x)\}$ is a cover of $X$ and $\cup\left\{U^{-1}(y): y \notin U(x)\right\}$ is disjoint from $U(x)$. It follows that $U(x)$ is $\mathscr{T}\left(\mathscr{N}^{-1}\right)$-closed. The proof of the corresponding result for $U^{-1}(x)$ follows in an analogous way.

Because the Nachbin quasi-uniformity of $(X, \sigma, \tau)$ is contained in the Pervin quasiuniformity, it is natural to consider when these quasi-uniformities coincide.

PROPOSITION 2.3. The Nachbin quasi-uniformity of $(X, \sigma, \tau)$ is $\mathscr{P}_{\sigma}$ if and only if $\tau$ is the Skula topology of $\sigma$.

PROOF. Suppose that $\tau$ is the Skula topology of $\sigma$. Then every $\sigma$-open set is $\tau$-closed and hence strictly regular. Hence the base $\mathscr{B}$ for $\sigma$ given in the proof of Proposition 2.1 is $\sigma$ itself and so $\mathscr{N}=\mathscr{V}=\mathscr{P}_{\sigma}$.

Now suppose that $\mathscr{P}_{\sigma}=\mathscr{N}$. Then $\mathscr{T}\left(\mathscr{P}_{\sigma}^{*}\right)=\mathscr{T}\left(\mathscr{N}^{*}\right)=\tau$ and $\mathscr{T}\left(\mathscr{P}_{\sigma}^{*}\right)$ is the Skula topology of $\sigma$.

COROLLARY 2.2. The quasi-proximities $\delta_{\mathscr{P}_{\sigma}}$ and $\delta_{\mathscr{N}}$ agree if and only if $\tau$ is the Skula topology of $\sigma$.

In light of Corollary 2.2, the last result of this section is somewhat surprising.

PROPOSITION 2.4. Let $A$ and $B$ be $\tau$-closed sets. Then $A \delta_{\mathscr{P}_{\sigma}} B$ if and only if $A \delta_{\mathscr{N}} B$. 
PROOF. Since $\mathscr{N} \subseteq \mathscr{P}$, if $A \delta_{\mathscr{P}_{\sigma}} B$, then $A \delta_{\mathscr{N}} B$. Suppose that $A \delta_{\mathscr{N}} B$. Then for each $U \in \mathscr{N}, U \cap A \times B \neq \emptyset$ and we must show that $A \cap \operatorname{cl}_{\sigma} B \neq \emptyset$. If $\leq_{\sigma} \cap A \times B=\emptyset$, then $X \times X-A \times B \in \mathscr{N}$ - a contradiction. Thus there exists $x \in A$ and $y \in B$ such that $x \leq_{\sigma} y$ and it follows that $A \cap \mathrm{cl}_{\sigma} B \neq \emptyset$.

EXAMPLE. We show here that in general for a bi-Stonian space $(X, \sigma, \tau)$, the Skula modification $S k(\sigma)$ of $\sigma$ is not $\tau$. Consider the chain $L=[0, \alpha]$ where $\alpha$ is an initial ordinal. It is readily seen that its Boolean extension, $B_{L}$, resides in $\mathscr{P}([0, \alpha))$ and that $L$ is embedded in $B_{L}$ by mapping $\beta$ to $[0, \beta)$ for $0 \leq \beta \leq \alpha$. For the ground set we take $X=\prod B_{L}$, the Stone space of $B_{L}$, which has as points all ultrafilters in $B_{L}$, and, for $F \in L$, we denote by $\prod_{F}$ the collection of all points of $\prod B_{L}$ that contain $F$. We take the Stone topology for $\tau$, and for $\sigma$ we take the topology for which $\left\{\prod_{[0, \beta)}: \beta \in[0, \alpha]\right\} \cup\left\{\prod B_{L}\right\}$ is a base. Take $P=\bigcap_{n \in \mathbb{N}} \prod_{[n, \alpha)}$. Evidently $P$ is in $S k(\sigma)$, since each $\prod_{[n, \alpha)}$ is $\sigma$-closed. We show that $P \neq \prod_{\left[w_{0}, \alpha\right)}$, after which, by checking other cases, it is readily seen that $P \notin \tau$.

The set $\{[n, \alpha): n \in \mathbb{N}\} \cup\left\{\left[0, w_{0}\right)\right\}$ has the finite intersection property, so can be extended to an ultrafilter $\mathscr{F}$ in $B_{L}$, which clearly contains each $[n, a)$, but not $\left[w_{0}, \alpha\right)$. Hence $\mathscr{F} \in P$, but $\mathscr{F} \notin \prod_{\left[w_{0}, \alpha\right)}$, as required.

In the case where $\alpha=w_{0}$, the example is particularly simple, since then $B_{L}$ is just all finite or cofinite subsets of $\left[0, w_{0}\right)$.

\section{References}

[1] G. C. L. Brümmer, 'Initial quasi-uniformities', Nederl. Akad. Wetensch. Proc. Ser A 72: Indag. Math. 31 (1969), 403-409.

[2] P. Fletcher and W. F. Lindgren, Quasi-uniform spaces, Lecture Notes in Pure and Applied Math. 77 (Marcel Dekker, New York, 1982).

[3] L. Nachbin, Topology and order, Van Nostrand Mathematical Studies 4 (Van Nostrand, Princeton, 1965). Reprinted by Robert E. Kreiger Publishing Co., Huntington, 1976.

[4] C. W. Neville, 'A Loomis-Sikorski theorem for locales', Ann. New York Acad. Sci. 552 (1989), 99-108.

[5] S. Salbany, Bitopological spaces, compactifications and completions, Mathematical Monographs of the University of Cape Town 1 (Department of Mathematics, University of Cape Town, 1974).

[6] L. Skula, 'On a reflective subcategory of the category of all topological spaces', Trans. Amer. Math. Soc. 142 (1969), 37-41.

Mathematics Department

Virginia Polytechnic Institute

Blacksburg VA 24061

USA

e-mail: pfletche@math.vt.edu
Mathematics Department University of Cape Town Rondebosch 7700 South Africa e-mail: jfrith@maths.uct.ac.za 
Mathematics Department

Southern Illinois University

Carbondale IL 62901

USA

e-mail: hunsaker@math.siu.edu
Mathematics Department University of Cape Town

Rondebosch 7700

South Africa

e-mail: schauert@math.uct.ac.za 